\title{
Visualizing the Maturing Global API Ecosystem
}

\author{
Saeid Heshmatisafa \\ Tampere University \\ saeid.heshmatisafa@tuni.fi
}

\author{
Jukka Huhtamäki \\ Tampere University \\ jukka.huhtamaki@tuni.fi
}

\author{
Marko Seppänen \\ Tampere University \\ marko.seppanen@tuni.fi
}

\begin{abstract}
Platform boundary resources are prominent digital strategy tools for companies to disclose business assets to partners and consumers. However, the platform boundary resources of API ecosystems are dependent on API usage and characteristics as well as the social environment around them. This study focuses on the global API ecosystem guided by two assumptions: 1) geographic proximity is distorted, and the global API ecosystem is decentralized in a digitally connected world, and 2) open co-innovation is somewhat prone to geographic nearness and centralized. A data-driven network analysis was utilized to depict the global and regional geolocation of more than 22,000 APIs and 6,000 mashups. First, the global locations of API providers were examined. Next, the connectivity of API mashups at global and regional levels was studied. The findings suggest that APIs are distributed globally, and the API ecosystem has moved from emerging to mature; however, the distribution is skewed towards entrepreneurial regions in North America. Finally, the theoretical and managerial implications are discussed.
\end{abstract}

\section{Introduction}

Platforms exist in a variety of industries, especially in high-tech businesses, for decades [1]. As platforms have become pervasive, scholars and practitioners have focused more on the phenomenon, resulting in several definitions. At a high level, platforms can be defined as enablers of value co-creation among different parties, such as complementors and consumers [2]. From a technical standpoint, maintaining and supporting such interaction between different parties requires careful management and the design of regulations. This can be done through boundary resources such as Software Development Kits (SDKs), and Application Programming Interfaces (APIs).

APIs are boundary resources that allow platform owners to control the ecosystem and delegate design capabilities to developers [3], [4]. Hence, these boundary resources are control points that regulate the relationship between the complementors and consumers. Studies on digital platforms suggest that, among boundary resources, APIs are the most popular instrument for designing the architecture and governance of digital platforms [5]-[7]. The omnipresence of APIs implies that APIs have become a popular digital strategy tool [8]. Hence, APIs are a key enabler of digital transformation and innovation. In essence, APIs allow companies to expose digital assets to third party consumers in order to build innovative solutions on top of the existing ones [9]. Implementation of APIs enables firms to gain significant leverage from features such as agile development, scalability, and network effects; however, many overlook this digital strategy tool. Iyer [9] has suggested that APIs are the future of interfirm collaboration. Furthermore, it is believed that companies are becoming more and more hyperconnected; thus, firms that aim to sustain in the market are required to adopt the development of API strategies [10]. Thus, this research attempts to shed light on the global collaboration and geographic proximity of the API ecosystem.

This study is a replication of prior studies on the use of APIs in the global ecosystem with some modifications in methodology and result analysis. There are two common assumptions in the domain of the global API ecosystem. First, in the digital era, it is assumed that geographic proximity is distorted, and the global API ecosystem is decentralized. Alternatively, open co-innovation is rather prone to geographic nearness and centralized. To address these assumptions, the study is done by exploring the global footprint of APIs. More precisely, the co-creation and reciprocity of the API ecosystem are investigated by mapping and visualizing the geolocations of API providers. Such geographic locations denote innovative sources of successful new digital product development and partnership. The results are narrowed to the top entrepreneurial global startup ecosystems [11]. 
Moreover, the outcomes extend the literature on the topology of the API ecosystem and digital innovation strategy [3], [12] as well as yielding categorical relationships between the APIs [10].

\section{Related Work}

\subsection{APIs and Mashups}

An API is a protocol that allows two computer programs to interconnect with one another over a network by using a common bilateral language [13]. Therefore, APIs can be defined as a medium to expose unique features (i.e., products, services, and results) to potential consumers (i.e., connect users, partners, communities, end-users, and developers) in a controlled, managed manner.

Initially, APIs were considered to lie in the domain of developers as a key part of web-centric development to accelerate delivery and enhance the robustness as well as the quality of the infrastructure [14]. However, APIs are not just gateways and technical artifacts. Currently, this boundary resource is at the center of digital platform ecosystems, enabling platform-based innovation between complementors and consumers [8].

The introduction of Service-Oriented Architecture (SOA) in the 2000s provided an opportunity for businesses to establish business-to-business relationships using standard interfaces and a Simple Object Access Protocol (SOAP) [15]. The concept of SOAP was further expanded to incorporate web-based technologies such as representational state transfer (REST) [16]. The RESTful approach paved the way for applying the HTTP protocol to construct and publish resources over the Internet and foster the culture of reusability by adapting create, read, update, and delete interfaces.

Mashups allow the orchestration of multiple resources and methods to be "consumed". Generally, API mashups are created by integrating resources from one-to-many providers to develop a new solution [17]. Therefore, it can be argued that mashup occurs within the API economy strategy of numerous API providers offering multiple methods to different kinds of API consumers. There are three primary levels of mashup within the industry [8], [17]. First, the raw data can be cultivated from one-to-many sources for internal or external use. Second, application logic can be compiled from existing components in a way that conceals the details of the source to provide a seamless experience. Third, many companies, such as Twitter, Facebook, and eBay, develop such components at little or no cost for third-party users to develop composite applications.

\subsection{Digital Platforms and the role of APIs}

Digital platforms have reshaped the market by enabling companies to exchange digital goods and services via microservices. Digital platforms serve as a foundation upon which complementors and consumers interact with one another in a convenient and coherent manner [1], [18]. Scholars offer many definitions for this phenomenon. Nonetheless, digital platforms can be defined as an "editable" and "reprogrammable" architecture of related standards that allows the owners to expand functionality and embed complementary modules for third-party users in order to construct a modular substitution of complementary assets [19], [20]. In this regard, incumbents have developed a digital platform to unite the scattered know-how and resources of various firms and connect users with producers.

Digital platforms are empowered by boundary resources such as SDKs and APIs [5]. APIs function as a boundary resource to match digital assets to users based on control arrangements and contractual agreements through personalization [18]. In this regard, APIs can expose business assets to external consumers and enable firms to reach beyond organizational boundaries to co-create business values. Consequently, API adaptation increases sales, net income, and market capitalization, as well as decreases operational costs [21].

Often the development of new business models and disruption of industries is attributed to technological and digital innovation [22]. However, new technologies per se do not disrupt one industry or enable another; it is the strategic implication of these new technologies that shifts the competitive landscape, excluding the adverse impacts of natural disasters [23], [24]. The API economy was first reviewed and visualized by Weiss and Gangadharan [12]. A study by Evans and Basole [3] showed that Amazon's entire business is constructed through APIs. Similarly, 90 percent of Expedia's, and 60 percent of eBay's revenues are generated via APIs [15]. In contrast, Walmart has a limited number of APIs [3]. Thus, it can be argued that APIs, particularly public APIs, are becoming strategic tools for numerous businesses [8]. Moreover, the structure of APIs supports the design of multi-sided business models that result in the onboarding of manifold users, and the creation of positive and negative network effects [25].

\section{Methodology}

\subsection{Empirical case description}

Following previous studies [3], [10], we have explored the geography of the global API ecosystem 
using a data-driven methodology. The research was carried out utilizing a data-driven approach by applying visual network analytics based on the Ostinato Model [26]. The exploration was based on an empirical case of API co-use in mashups. Following the data collection methodologies of Weiss and Gangadharan [12], Evans and Basole [3], and Basole [10], the process was initiated by gathering a list of mashups, APIs, and API versions to build a linkage of co-use between each API entity. Next, a commonly used method in scientometrics known as co-citation analysis [27] was utilized on collected data sets to construct an analytical network of the interconnections of individual APIs.

To incorporate the geolocation layer into the analysis, APIs were connected to providers. This enabled the identification of the geographical location of each individual API provider and further addressed the latitude and longitude coordinates to establish entrepreneurial and global regions.

Afterward, the accumulated data sets were analyzed and visualized. First, a global base map of the distribution of API providers was created. Second, the relationship between the residential sites of each entrepreneurial region was demonstrated. Third, the statistical metrics of the internal connectivity of the entrepreneurial locales were presented. Fourth, the contribution of individual entrepreneurial regions in different sectors was reviewed. Fifth, a comparison to prior studies was made.

\subsection{Data}

The investigation of the geographical API ecosystem as a network was done by examining the leading API and mashup repository, ProgrammableWeb. Over the past fifteen years, this website has maintained a comprehensive list of API and mashups, including information on API providers and their mashup use (Figure 1). The data gathering process followed a custom-made procedure that crawls individual APIs, API versions, and mashup pages. The accumulated data set, obtained at the beginning of 2021, consists of a total of 22,074 APIs, 22,735 API versions, and 6,438 mashups.

In order to locate API providers, APIs, and API versions were aggregated with a publicly available data set of over a million SMEs, Crunchbase. Consequently, a four-step process was undertaken to acquire the unique attributes of each API and match them with Crunchbase records. First, all of the relevant URLs such as the API portal/homepage, endpoint, and developer support were collected and processed to extract and map them by domain. Second, to handle URL redirecting services such as bit.ly, a request was sent to each shortened link, and the final URL was stored. Third, a tailor-made
Levenshtein Distance method was employed to manage the use of third-party platform account links by means of homepages such as GitHub, Apiary, Mashape, ReadMe, and ApiDocs. Thus, the Levenshtein distance was applied to company domains and names of related companies to measure the similarities and ultimately select the best match. Finally, FuzzyWuzzy version 0.18, a standard Levenshtein distance Python package, was invoked to address the use of the commercial name in one data set and the formal company name in the other. This way, we were able to map the location of a total of 13,723 APIs successfully.

In addition, we turned to the Countryinfo library to link API destinations to their corresponding ISO 31662 country codes. Subsequently, the three-letter country codes were employed in parallel with the GeoPy package to resolve the geolocation of APIs. Latitude and longitude coordinates were used to classify the locations of the API providers into two levels: city and global region (North America, Europe, and East Asia \& Pacific). Ultimately, the Startup Genome data set of the Global Startup Ecosystem 2020 was used to build entrepreneurial regions.

\subsection{Visualization process}

The network creation process was managed by tailor-made Python scripts. The scripts encompass several Python packages. For instance, Pandas and NumPy libraries were imported to transform, process, clean, and vectorize the data sets. In addition, NetworkX [28] was applied to compile and analyze the network. During the visualization process, the relevant entities were filtered to create nodes and edges. The nodes represent the entrepreneurial regions, while the node size portrays the regional bridge score, also known as betweenness centrality. The betweenness centrality is a metric to demonstrate the connecting role of each node in the ecosystem. In other words, the larger the node, the higher the bridging score incurred. The edge thickness corresponds to the total number of mashups using APIs from a pair of entrepreneurial regions. Moreover, the node and edge colors represent global regions.

Once the network graph files were serialized, Gephi, an open source network exploration and analysis software [29], was used to visualize the network of entrepreneurial regions in the API co-use ecosystem. The network was laid out using a combination of three force-driven algorithms. The Force Atlas algorithm was executed to distribute the regions by node gravity. Afterward, Noverlap and Label Adjust algorithms were applied to the network for better readability. The remaining figures were produced using the visual analytics platform Tableau and the Plotly package. 


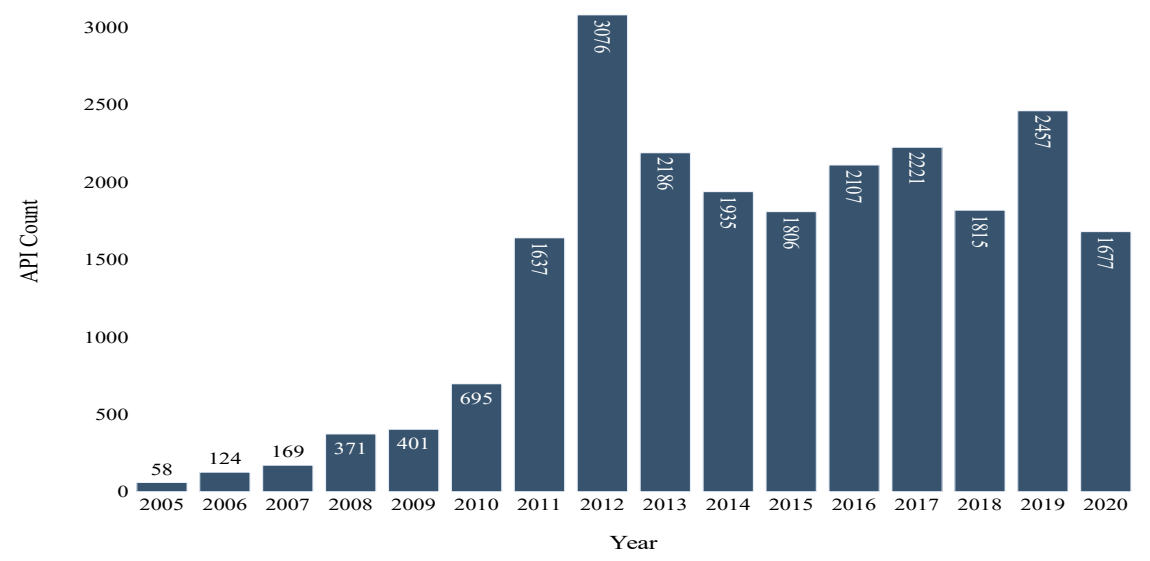

Figure 1. ProgrammableWeb API growth

\section{Analysis and results}

Figure 2 depicts the API providers' locations and the size associated with the frequency of APIs available over states/provinces on a global scale. With 3,181 APIs, Silicon Valley remains the leading API provider of the global API ecosystem. Compared to the previous study in $2016(n=1623)$, this number has approximately doubled, with a growth rate of $51 \%$. In second place, New York, with a 52\% growth rate, provides over 881 APIs. London $(n=683)$ is the third leading global API provider and ranks first in Europe. It is worth noting that London, with a rate of growth of $40 \%$ has surpassed
Washington DC and moved from fourth place to third. In fourth to sixth place, Boston, Washington DC, and Seattle contribute to this ecosystem by providing 521, 513, 482 APIs, respectively. In addition, compared to the previous study, Tampa, Singapore, Stockholm, and Zurich are new actors in the global API ecosystem. Finally, Singapore has become the leading API provider in Asia.

The network analysis of entrepreneurial locales provides a valuable insight into API co-use between each region. The network shown in Figure 3 illustrates the individual regions (nodes) and their corresponding API connectivity (edge) (i.e., the number of mashups,

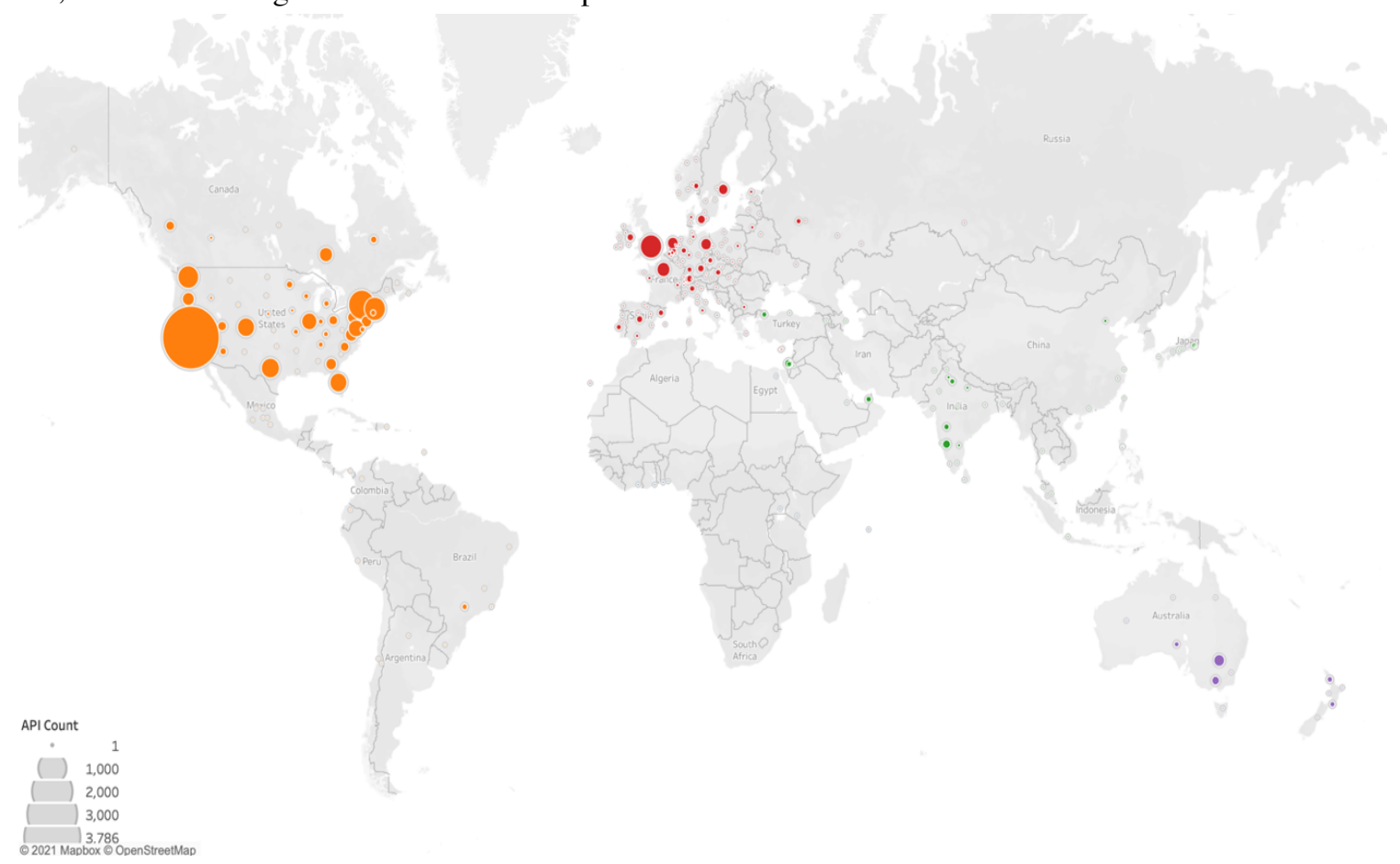

Figure 2. Global API locations 
Table 1. Summary of regional API ecosystem statistics

\begin{tabular}{|c|c|c|c|c|c|c|c|c|c|c|c|c|}
\hline & Region & $\begin{array}{c}\text { Global } \\
\text { Startup } \\
\text { Index }\end{array}$ & Diff & $\begin{array}{c}\text { Global } \\
\text { Emerging } \\
\text { Startup } \\
\text { Index }\end{array}$ & $\begin{array}{l}\text { API } \\
\text { Count }\end{array}$ & $\begin{array}{c}\text { Mashup } \\
\text { Count }\end{array}$ & $\begin{array}{c}\text { Region } \\
\text { Bridge } \\
\text { Score }\end{array}$ & Nodes & Edges & Density & $\begin{array}{l}\text { Average } \\
\text { Degree }\end{array}$ & $\begin{array}{l}\text { Average } \\
\text { Clustering }\end{array}$ \\
\hline 1 & Silicon Valley & 1 & 0 & - & 3181 & 6833 & 0.422 & 310 & 1486 & 0.031 & 9.587 & 0.508 \\
\hline 2 & New York & 2 & 0 & - & 881 & 376 & 0.040 & 64 & 45 & 0.022 & 1.406 & 0.150 \\
\hline 3 & London & 2 & 1 & - & 683 & 274 & 0.021 & 39 & 6 & 0.008 & 0.308 & 0.000 \\
\hline 4 & Boston & 5 & $(-1)$ & - & 521 & 40 & 0.007 & 20 & 0 & 0.000 & 0.000 & 0.000 \\
\hline 5 & $\begin{array}{l}\text { Washington } \\
\text { DC }\end{array}$ & 11 & $(-6)$ & - & 513 & 86 & 0.011 & 30 & 3 & 0.007 & 0.200 & 0.000 \\
\hline 6 & Seattle & 9 & $(-3)$ & - & 482 & 883 & 0.059 & 60 & 86 & 0.049 & 2.867 & 0.415 \\
\hline 7 & $\begin{array}{l}\text { Los Angeles- } \\
\text { Orange County }\end{array}$ & 6 & 1 & - & 340 & 146 & 0.010 & 21 & 2 & 0.010 & 0.190 & 0.000 \\
\hline 8 & Denver/Boulder & 24 & $(-16)$ & - & 338 & 59 & 0.001 & 14 & 1 & 0.011 & 0.143 & 0.000 \\
\hline 9 & Chicago & 14 & $(-5)$ & - & 272 & 57 & 0.005 & 19 & 1 & 0.006 & 0.105 & 0.000 \\
\hline 10 & Tampa & - & - & 24 & 243 & 5 & 0.000 & 2 & 0 & 0.000 & 0.000 & 0.000 \\
\hline 11 & Paris & 13 & $(-2)$ & - & 199 & 22 & 0.002 & 9 & 0 & 0.000 & 0.000 & 0.000 \\
\hline 12 & Singapore & 17 & $(-5)$ & - & 183 & 27 & 0.002 & 8 & 2 & 0.071 & 0.500 & 0.000 \\
\hline 13 & Austin & 19 & $(-6)$ & - & 169 & 19 & 0.002 & 9 & 3 & 0.083 & 0.667 & 0.333 \\
\hline 14 & Amsterdam & 12 & 2 & - & 168 & 10 & 0.000 & 6 & 0 & 0.000 & 0.000 & 0.000 \\
\hline 15 & Portland & - & - & 15 & 164 & 32 & 0.000 & 8 & 0 & 0.000 & 0.000 & 0.000 \\
\hline 16 & Toronto & 18 & $(-2)$ & - & 154 & 21 & 0.013 & 9 & 0 & 0.000 & 0.000 & 0.000 \\
\hline 17 & Philadelphia & - & - & 8 & 154 & 8 & 0.001 & 5 & 0 & 0.000 & 0.000 & 0.000 \\
\hline 18 & Sydney & 27 & $(-9)$ & - & 148 & 6 & 0.000 & 6 & 3 & 0.200 & 1.000 & 0.500 \\
\hline 19 & Berlin & 16 & 3 & - & 137 & 36 & 0.002 & 4 & 0 & 0.000 & 0.000 & 0.000 \\
\hline 20 & Atlanta & 23 & $(-3)$ & - & 116 & 36 & 0.002 & 5 & 0 & 0.000 & 0.000 & 0.000 \\
\hline 21 & Stockholm & 19 & 2 & - & 106 & 6 & 0.000 & 4 & 0 & 0.000 & 0.000 & 0.000 \\
\hline 22 & Dallas & 31 & $(-9)$ & - & 103 & 11 & 0.001 & 4 & 0 & 0.000 & 0.000 & 0.000 \\
\hline 23 & Tel Aviv & 6 & 17 & - & 98 & 7 & 0.000 & 4 & 0 & 0.000 & 0.000 & 0.000 \\
\hline 24 & Vancouver & 25 & $(-1)$ & - & 91 & 5 & 0.000 & 3 & 0 & 0.000 & 0.000 & 0.000 \\
\hline 25 & Salt Lake City & 31 & $(-6)$ & - & 82 & 9 & 0.000 & 3 & 0 & 0.000 & 0.000 & 0.000 \\
\hline 26 & Copenhagen & 36 & $(-10)$ & - & 75 & 2 & 0.000 & 1 & 0 & 0.000 & 0.000 & 0.000 \\
\hline 27 & San Diego & 21 & 6 & - & 72 & 82 & 0.001 & 10 & 3 & 0.067 & 0.600 & 0.000 \\
\hline 28 & Zurich & - & 11 & 3 & 65 & 9 & 0.000 & 4 & 0 & 0.000 & 0,000 & 0.000 \\
\hline 29 & Dublin & 36 & $(-7)$ & - & 62 & 3 & 0.000 & 2 & 0 & 0.000 & 0.000 & 0.000 \\
\hline 30 & Raleigh & - & - & - & 59 & 11 & 0.001 & 6 & 0 & 0.000 & 0.000 & 0.000 \\
\hline
\end{tabular}




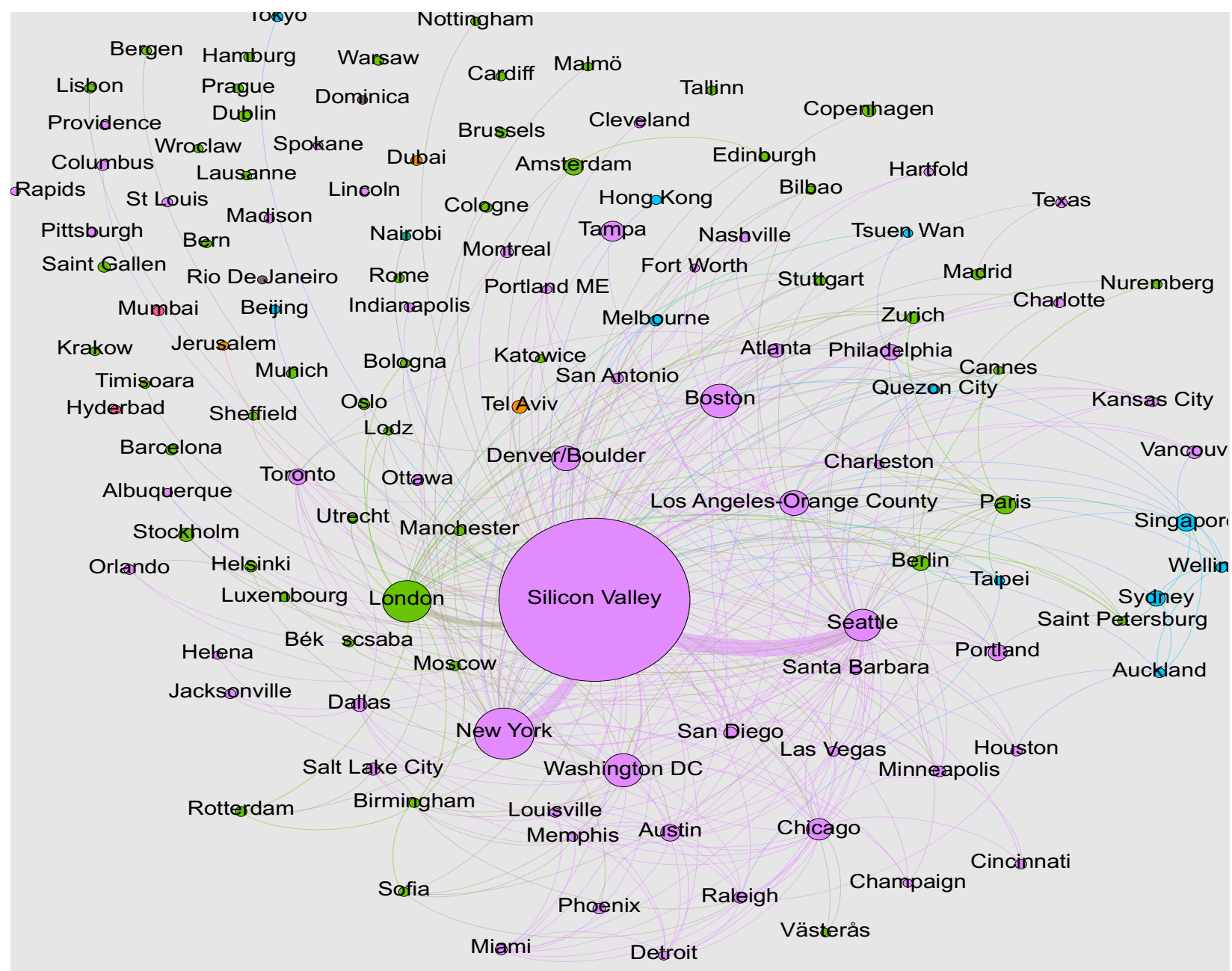

Figure 3. Network of API co-use between entrepreneurial regions

between regions). It is evident that Silicon Valley is the primary API provider in the global API ecosystem, which attracts and feeds various regions. Moreover, the thickness of edges represents the mashup volume driven from a region. There is a significant collaboration between Silicon Valley as the central source and New York, London, and Seattle. Compared to the previous study, the co-use between the above-mentioned regions has grown considerably. For instance, in the previous study, we could barely see any strong cooperation between Silicon Valley and New York; whereas, currently, after Seattle, New York has the strongest mashup with Silicon Valley. In any case, North America hosts the mainstream of entrepreneurial regions.

Table 1 presents some descriptive quantitative metrics of the entrepreneurial regions to supplement visual analysis. The number of available APIs of individual regions and their associated mashup frequency are provided. To present the internal connectivity of each region, a set of metrics have been computed. The density reveals the number of potential connections between actors of the ecosystem compared to actual connections. The average node degree refers to the average proportion of APIs that an API is connected to. The clustering coefficient discloses the abundance of connected triangles (nodes) that tend to cluster together. When the linkages between nodes are dense, the cluster coefficient is relatively high. This means that when the region's "friends" collaborate with one another, it increases the cluster coefficient. In addition, the total number of nodes and edges are given.

From Table 1, it is evident that Silicon Valley, as the leading API provider and a vibrant source of the ecosystem, has the greatest clustering coefficient. This can also be observed by looking at Figure 3. In contrast, Seattle, Sydney, and Austin, with a relatively lower number of APIs compared to Silicon Valley, achieved approximately the same degree and clustering 
coefficient. This may imply that the APIs provided by Seattle, Sydney, and Austin are merged with various other APIs, which increased their average degree and clustering.

Additionally, the entrepreneurial regions have been sorted by the frequency of available APIs. Afterward, the difference between the entrepreneurial regions' ranks and the Global Startup Index was calculated by filling the missing values with the mean and the difference between the two sets of ranks. The difference between the two ranks for Denver/Boulder, Tel Aviv, and Copenhagen are worthy of note. Denver/Boulder, with a substantial number of APIs - ranked eighth in our study - is placed 24th in the Global Startup Index report. The same contrast is valid in the case of Copenhagen. However, in contrast, Tel Aviv is in sixth place based on the Global Startup Index, whereas it is in 24th place in our global API ecosystem. Tampa, Portland, Philadelphia, Zurich, and Raleigh, with a significant number of APIs, are not included in the Global Startup Index; however, the first four regions are acknowledged as emerging startup regions.

The APIs represented on ProgrammableWeb are classified into 427 unique categories. The highest numbers of applications of APIs are primarily in finance, tools, payment, messaging, and e-commerce sectors, ranging between 600 and over 1000. Enterprise, social, mapping, science, and government are similarly notable sectors, each including between 300 and 400 APIs per sector.

To explore the relationship between the regions and industry sectors, we plotted a productivity heatmap of entrepreneurial regions over the top 10 categories (Figure 4). In order to present visual cues, the data has been normalized in the range of 0 to 1 (Min-Max Scalar). Finally, the figure is color-coded with higher saturation, indicating the greater concentration of individual regions in a specific category.

The top five entrepreneurial regions (Silicon Valley, New York, London, Boston, Seattle) are the only regions that contribute to all ten categories. Silicon Valley's contribution is scattered across all sectors, with the highest focus on financial APIs and the least on governance APIs. New York follows the same trend as Silicon Valley. In contrast, governance APIs are the key sector in Washington DC and Portland.

In Europe, the entrepreneurial regions of London, Amsterdam, Copenhagen, and Zurich primarily provide payment APIs. The exceptions are Paris, Berlin, and Stockholm. In Paris, the enterprise sector holds first place, followed by tools in second place. There appear to be somewhat equal contributions in the sectors of finance, messaging, and social, whereas in Berlin and Stockholm, eCommerce APIs have the first position, and payments the second. It is worth mentioning that, in Berlin, productivity between the tools and eCommerce sectors is roughly even.

The findings do not suggest any patterns between the representatives of Asia: Singapore and Tel Aviv. Singapore, as the new actor in the global API economy and leading the field in Asia, is specialized in the financial sector. API providers from Tel Aviv are more invested in mapping and messaging.

To bridge the current study with the prior one we plotted Figure 5. Thus, we have a clear comparison between the results of the studies in 2016 and 2021. On

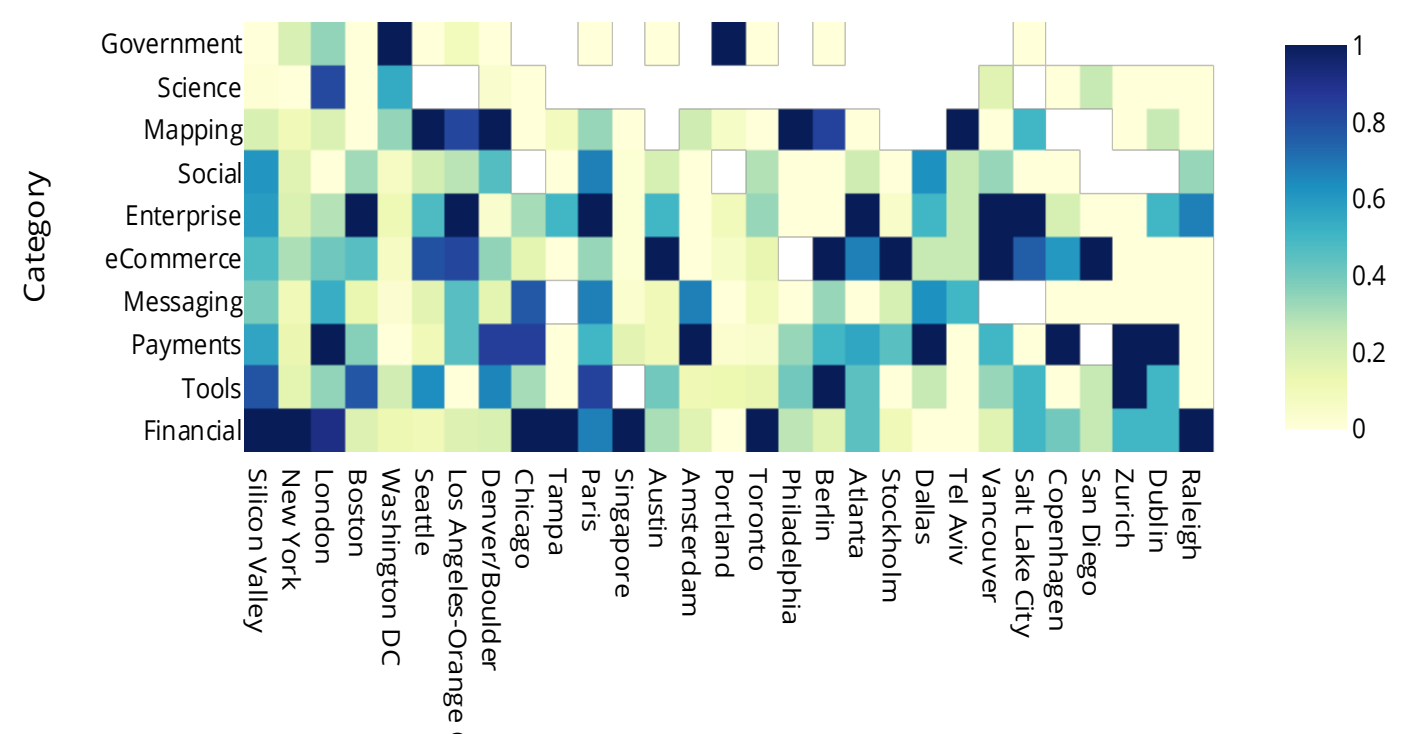

Figure 4. Entrepreneurial regions by category. 


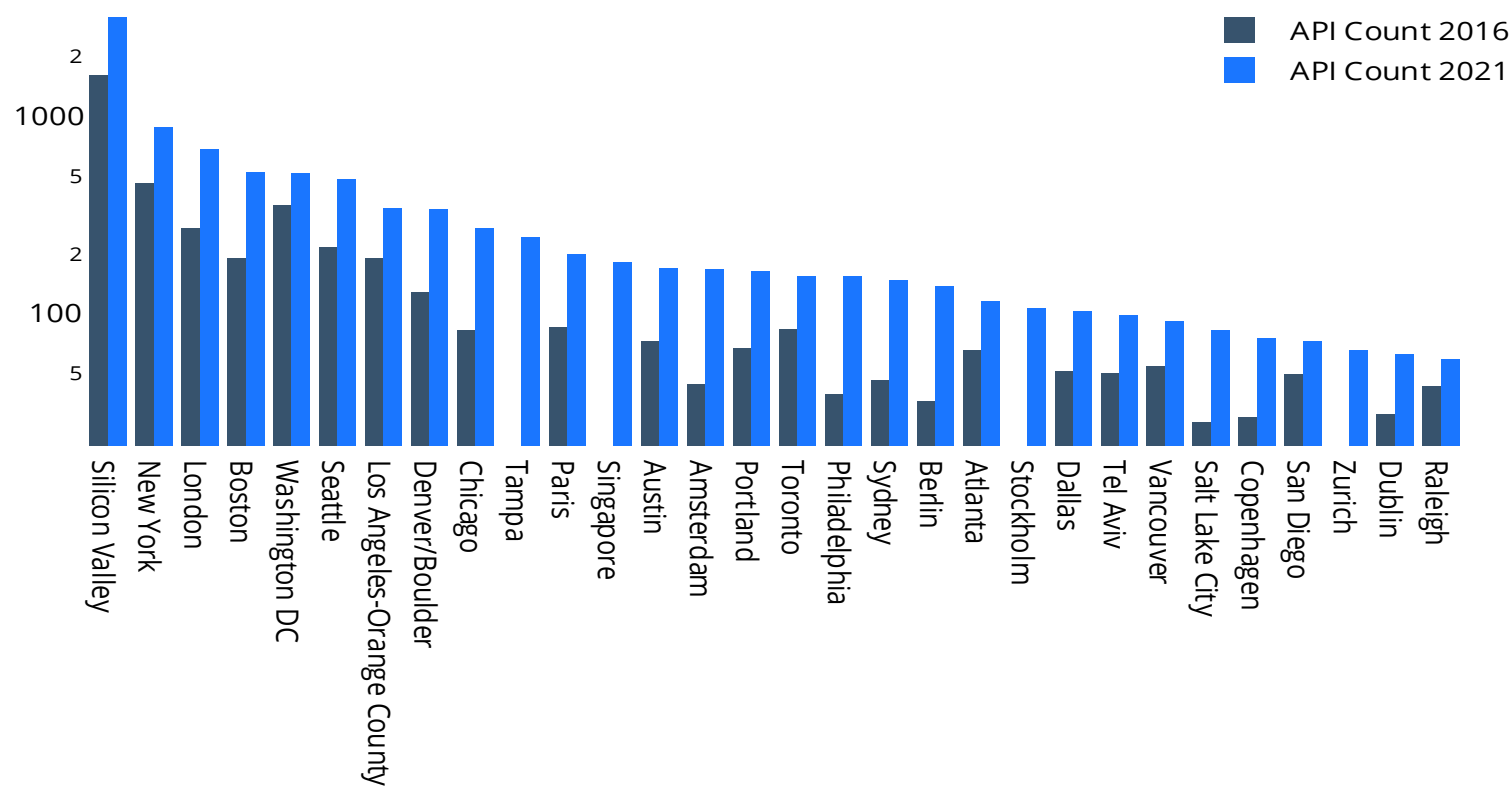

Figure 5. Global API ecosystem comparison between 2016 and 2021.

average, all entrepreneurial regions have a growth rate of approximately $44 \%$. Furthermore, the number of APIs has risen steadily among the top 30 entrepreneurial regions. This indicates that the importance of the phenomenon has not deteriorated and is still growing.

Growth percentages are consistent overall across the top entrepreneurial regions. However, considerable growth can be seen in the Amsterdam, Berlin, Copenhagen, Stockholm, and Zurich regions. This signifies that Europe is finding its way to competing in the API ecosystem. In contrast, the APIs originating in Washington DC did not follow quite the same trend compared to the rest of the regions. Moreover, the outcomes of the 2021 study suggest that the productivity curve for the top 29 regions is flattening. Thus, we expect to witness a shift toward a more balanced global distribution of API ecosystems in future. Presumably, the decentralization of API providers duo to the emergence of new entrants.

\section{Discussion}

Our research on API geolocations is founded on the notion that, in the field of the platform economy, APIs are innovative types of boundary resources. Digitalization indicates that physical and geographic borders are irrelevant [30]. Conversely, the results of the study suggest that the colocation of APIs plays a vital role in developing young businesses. Thus, we reject the first assumption of the decentralization of API consumption at the global level. It appears that North America, with the substantial contribution of Silicon
Valley, remains the hub of the API ecosystem. However, centralization has not become faster, as the growth percentages of the top locations are roughly the same as earlier. Nonetheless, there is no evidence that the global API ecosystem has progressed towards decentralization. In other words, no substantial decentralization is taking place.

Moreover, Table 1 and Figure 5 demonstrate that New York has grown considerably as an API provider compared to the previous study. Hence, we anticipate that Silicon Valley is losing its vitality due to high prices, migration of high-tech companies to New York, and issues regarding counterforces to centralization. Nevertheless, this assumption requires further investigation.

In terms of data-driven methodology, with the perpetual progression of content privacy extremists, data collection and analysis are becoming less and less feasible. Such movements hamper and weaken studies on platform ecosystems. Consequently, the data collection process of ProgrammableWeb confronts this by anti-crawling techniques, which pose challenges for our study. Moreover, an API catalog that does not itself provide an API presents an interesting paradox. Knowing that anti-scraping techniques are one way to handle bad bots and manage traffic, we would propose that platform owners consider the development of the boundary resources of API with respect to European GDPR.

New API offerings are constantly entering the market, either novel solutions or a combination of existing APIs. Hence, if an API provider retires an API, restrict access, or change the monetization strategy, 
developers have the autonomy to switch to alternative products. Whereas, in the case of API platforms, developers are more reliant on the platform owner. Thus, for an API provider establishing a network with an ecosystem is useful for underscoring the importance of interdependencies between different agents in the innovation process and across the value chain, which simultaneously promotes survival and success.

It worth noting that, the Public Open API ecosystem differs from the traditional platform ecosystem. In traditional platform ecosystems, owner(s) or key partners can utilize boundary resources to govern and select potential partners for co-creation. However, in Public Open API ecosystems, the borders for entrants are blurred, and the growth of API consumers and partnerships takes a more organic approach. Nevertheless, both approaches have their pros and cons, which is beyond the scope of this research.

\section{Conclusion}

This research makes several contributions. First, we contributed to the scant empirical body of knowledge on API research by depicting the status of the global API ecosystem in 2021 and comparing our findings to the 2016 results. Second, we reproduced the analysis of the global API ecosystem by solving the structural changes in ProgrammableWeb regarding anti-crawling and API versioning. Third, our outcomes advocate further research on API ecosystem orchestration, design, and policymaking.

The key insights for policymakers are as follows: 1) Silicon Valley is being contested and challenged by New York; a plethora of services and products from Silicon Valley based companies are available via APIs which enable mashup with third-party APIs scattered across various geographic locations and categories. 2) New York is a major emergent region, primarily due to the migration of GAFA companies. 3) London is emerging as the central API hub in Europe, primarily due to FinTechs. However, Europe still provides fewer APIs and mashups than California alone. 4) Singapore, a newcomer to the API ecosystem, dominates the Asian market and is exclusively focused on the financial sector.

We recommend that API providers innovate in API design, documentation, and pricing strategy to address the scattered distribution of this boundary resource. Based on our knowledge, on average a single application consumes approximately 18 APIs. An API is a product designed by developers for developers; thus, careful API design and documentation can help firms with API recognition. In addition, physical interaction between API developers may not be essential; however, co-location in the development phase can be beneficial in context setting and fine-tuning.

Moreover, APIs do not merely offer greater flexibility and agility; they allow firms to attract and connect with more partners and customers. The results demonstrate that the API has surpassed its original purpose as a "technical asset" and become a major force of the economy. Open APIs enable companies to gain positive network effects along with spotting emerging trends, developing new products/services, and creating a digital economy. API integration accounts for more than half of B2B collaboration [31], and it is expected that more prospective partners will adopt lightweight APIs. Ergo, we encourage companies in Europe to seize such opportunities and reflect upon their level of contribution and participation in the API ecosystem.

The geographic spatial visualization of API providers signifies the digital path of innovation in the value chain of digital products and digital ecosystems. APIs standardize the interfaces between contributors and reduce the switching costs; hence, it can be argued that they change the competition and firms' possibilities to achieve lock-in by increasing flexibility and reducing cognitive path dependency.

There are several limitations associated with this study. Since API consumption is considered as a competitive intelligence in many companies, such data is not publicly available, which limited our studies to Public Open APIs. In addition, the growing number of APIs is boosting the development of new and young API directories and marketplaces such as RapidAPI.

Several possibilities for further research exist. First, detailed research on the internal API co-use structure of distinct regions will deepen our understanding of geographical proximity. We expect that internal API couse of distinct regions in the top five locations would be substantial. Second, conducting a study on the potential correlation between global API locations and venture capital investments may reveal valuable insight into the global API ecosystem. Third, it would be interesting to reveal the global level API ecosystem structure while maintaining a regional view. Fourth, research on the global API ecosystem with a different data set apart from company-provided data sources may provide a beneficial viewpoint. Fifth, a replication and update on firm-level API providers and their associated API couse would yield some insight. Finally, study of API ecosystem governance with consideration of the enforcement of GDPR legislation would aid policymakers.

\section{References}

[1] M. Dodgson, D. M. Gann, and N. Phillips, The Oxford handbook of innovation management. OUP 
Oxford, 2013.

[2] G. G. Parker, M. W. Van Alstyne, and S. P. Choudary, Platform revolution: how networked markets are transforming the economy and how to make them work for you. WW Norton \& Company, 2016.

[3] P. C. Evans and R. C. Basole, "Economic and business dimensions: Revealing the API ecosystem and enterprise strategy via visual analytics," Commun. ACM, vol. 59, no. 2, pp. 26-28, 2016, doi: $10.1145 / 2856447$.

[4] E. Von Hippel and R. Katz, "Shifting innovation to users via toolkits," Manage. Sci., vol. 48, no. 7, pp. 821-833, 2002.

[5] A. Tiwana, B. Konsynski, and A. A. Bush, "Research commentary-Platform evolution: Coevolution of platform architecture, governance, and environmental dynamics," Inf. Syst. Res., vol. 21, no. 4, pp. 675-687, 2010.

[6] A. Ghazawneh and O. Henfridsson, "Balancing platform control and external contribution in thirdparty development: the boundary resources model," Inf. Syst. J., vol. 23, no. 2, pp. 173-192, 2013.

[7] V. Dal Bianco, V. Myllärniemi, M. Komssi, and M. Raatikainen, "The role of platform boundary resources in software ecosystems: A case study," in 2014 IEEE/IFIP Conference on Software Architecture, 2014, pp. 11-20.

[8] J. Huhtamäki, R. Basole, K. Still, M. Russell, and M. Seppänen, "Visualizing the geography of platform boundary resources: The case of the global API ecosystem," 2017.

[9] B. Iyer, "To Predict the Trajectory of the Internet of Things, Look to the Software Industry," Harv. Bus. Rev., pp. 1-7, 2016, Accessed: Sep. 14, 2020. [Online]. Available: https://hbr.org/2016/02/topredict-the-trajectory-of-the-internet-of-things-lookto-the-software-industry.

[10] R. C. Basole, "Accelerating digital transformation: Visual insights from the API ecosystem," IT Prof., vol. 18 , no. 6, pp. 20-25, 2016.

[11] Startup Genome, "The Global Startup Ecosystem Report GSER 2020,” 2020.

[12] M. Weiss and G. R. Gangadharan, "Modeling the mashup ecosystem: structure and growth," $R \& d$ Manag., vol. 40, no. 1, pp. 40-49, 2010.

[13] D. Jacobson, D. Woods, and G. Brail, APIs: A strategy guide. "O'Reilly Media, Inc.," 2011.

[14] S. Heshmatisafa and M. Seppänen, "API Utilization and Monetization in Finnish Industries," in International Conference on Agile Software Development, 2020, pp. 23-31.

[15] M. Vukovic et al., "Riding and thriving on the API hype cycle," Commun. ACM, vol. 59, no. 3, pp. 3537, 2016.

[16] R. T. Fielding and R. N. Taylor, Architectural styles and the design of network-based software architectures, vol. 7. University of California, Irvine Irvine, 2000.
[17] S. Yu and C. J. Woodard, "Innovation in the programmable web: Characterizing the mashup ecosystem," in International Conference on ServiceOriented Computing, 2008, pp. 136-147.

[18] H. R. Varian, "Computer mediated transactions," Am. Econ. Rev., vol. 100, no. 2, pp. 1-10, 2010.

[19] M. De Reuver, H. Bouwman, and T. Haaker, "Business model roadmapping: A practical approach to come from an existing to a desired business model," Int. J. Innov. Manag., vol. 17, no. 01, p. 1340006, 2013.

[20] M. de Reuver, C. Sørensen, and R. C. Basole, "The digital platform: a research agenda," J. Inf. Technol., vol. 33, no. 2, pp. 124-135, 2018.

[21] S. Benzell, G. Lagarda, and M. W. Van Alstyne, "The Impact of APIs in Firm Performance," Bost. Univ. Questrom Sch.Bus. Res. Pap., no. 2843326, 2017.

[22] J. Karimi and Z. Walter, "The role of dynamic capabilities in responding to digital disruption: A factor-based study of the newspaper industry," $J$. Manag. Inf. Syst., vol. 32, no. 1, pp. 39-81, 2015.

[23] M. E. Porter, "Technology and competitive advantage," J. Bus. Strategy, vol. 5, no. 3, p. 60, 1985.

[24] G. C. Kane, D. Palmer, A. N. Phillips, D. Kiron, and N. Buckley, "Strategy, not technology, drives digital transformation," MIT Sloan Manag. Rev. Deloitte Univ. Press, vol. 14, no. 1-25, 2015.

[25] G. Parmentier and R. Gandia, "Redesigning the business model: from one-sided to multi-sided," $J$. Bus. Strategy, 2017.

[26] J. Huhtamäki, M. G. Russell, N. Rubens, and K. Still, "Ostinato: The exploration-automation cycle of user-centric, process-automated data-driven visual network analytics," in Transparency in social media, Springer, 2015, pp. 197-222.

[27] H. Small, "Co-citation in the scientific literature: A new measure of the relationship between two documents," J. Am. Soc. Inf. Sci., vol. 24, no. 4, pp. 265-269, 1973.

[28] A. Hagberg, P. Swart, and D. S Chult, "Exploring network structure, dynamics, and function using NetworkX," Los Alamos National Lab.(LANL), Los Alamos, NM (United States), 2008.

[29] M. Bastian, S. Heymann, and M. Jacomy, "Gephi: An Open Source Software for Exploring and Manipulating Networks," in ICWSM, 2009, pp. 361362, [Online]. Available:

http://www.aaai.org/ocs/index.php/ICWSM/09/pape r/view/154.

[30] A. Byrne, "Digital libraries: barriers or gateways to scholarly information?," Electron. Libr., 2003.

[31] M. Geene, R. Garrett, K. Lane, I. Mauny, and M. Boyd, "State of API Integration 2018," 2018. [Online]. Available: https://offers.cloudelements.com/hubfs/cld-2018-soai-final-2018.pdf. 